PHYSICAL REVIEW E 74, 031702 (2006)

\title{
Structural study of a bent-core liquid crystal showing the $B_{1}-B_{2}$ transition
}

\author{
Cesar Luis Folcia and J. Etxebarria \\ Departamento de Física de la Materia Condensada, Facultad de Ciencias,Universidad del País Vasco, Apartado 644, \\ 48080 Bilbao, Spain \\ J. Ortega \\ Departamento de Física Aplicada II, Facultad de Ciencias, Universidad del País Vasco, Apartado 644, 48080 Bilbao, Spain \\ M. B. Ros \\ Departamento de Química Orgánica, Facultad de Ciencias, Universidad de Zaragoza, 50009 Zaragoza, Spain
}

(Received 16 March 2006; published 1 September 2006)

\begin{abstract}
An experimental study of the $B_{1}-B_{2}$ transition is carried out in a bent-core liquid crystal. The study is essentially based on $\mathrm{x}$-ray measurements as a function of temperature. The $B_{1}-B_{2}$ transition is extremely unusual and implies a deep structural change from a columnar phase to a lamellar phase. We have found that the $B_{1}$ phase in our compound is similar to the so-called $B_{1}$ reversed phase, with an additional splay of the polarization in the columns. On approaching the $B_{2}$ phase the width of the cross section of the columns increases. The transition is clearly first order, with a large hysteresis though the enthalpy content is very small. A possible mechanism for the transition is briefly sketched.
\end{abstract}

DOI: 10.1103/PhysRevE.74.031702

PACS number(s): 64.70.Md, 61.10.Eq, 61.30.Eb

\section{INTRODUCTION}

Liquid-crystalline phases formed by bent-core molecules have been the object of intensive research over the last years. At least eight different phases (designated as $B_{1}-B_{8}$ ) have been officially identified, though this nomenclature is now being revised and most likely will be changed in the near future. The topic has been object of several review papers [1-5].

Among the eight referred mesophases the most extensively studied is the $B_{2}$ phase with all its variants [6] (ferroelectric and antiferroelectric, synclinic and anticlinic) followed by the columnar $B_{1}$ phase. In a great amount of material families, with the same core and different terminal chains, it has been found that on increasing the chain length the phase sequence $B_{6}-B_{1}-B_{2}$ takes place [7-9]. The $B_{2}$ and $B_{6}$ phases are single-layer and intercalated smectics, respectively, whereas the $B_{1}$ phase is a smectic-frustrated intermediate structure with a two-dimensional modulation. Accordingly, the above sequence has been roughly explained in terms of the different degree of overlap between cores and chains in the different structures (maximum in the $B_{6}$ phase and minimum in the $B_{2}$ phase). Usually the materials present only one of the three referred phases though sometimes the $B_{1}-B_{6}$ dimorphism is also found [10]. However, the $B_{1}-B_{2}$ dimorphism (both on heating and cooling) has been reported for very few compounds [11-14]. In some cases $[13,14]$ it has been found that a strong electric field applied to the $B_{1}$ phase can also induce the transition to $B_{2}$ or to another unidentified mesophase [15].

The peculiarity of the $B_{1}-B_{2}$ transition is not surprising because, in principle, it would suppose a drastic change in the structure of the material from columnar to lamellar. The mechanism of the transition is not evident at all, and thus it seems of interest to analyze this question in some detail. Part of the problem is the structure of the $B_{1}$ phase itself. Up to now several models for the molecular arrangement in the $B_{1}$ phase have been proposed in the literature [5,16-20]. Probably most of them are correct but, however, several structures with different symmetry groups are being designated under the common name of $B_{1}$. It is then interesting to identify what $B_{1}$ phase can give rise to a $B_{2}$ phase and how the transition takes place.

In this work we present an experimental study of one the compounds of Ref. [13] around its $B_{1}-B_{2}$ transition. The investigation is based on $\mathrm{X}$-ray diffraction and textures observation. The chemical structure and phase sequence are shown in Fig. 1. We present an analysis of the $B_{1}$ structure as a function of temperature. Based on these results a structural model for the $B_{1}$ phase of the compound is proposed, and the character and mechanisms of the transition are discussed.

\section{EXPERIMENTAL}

X-ray measurements were carried out on nonoriented samples by means of a small-angle goniometer with a hightemperature attachment. The materials were introduced in the isotropic phase into Lindeman capillaries of diameter $0.5 \mathrm{~mm}$. The $\mathrm{x}$-ray intensity was detected with a linear position-sensitive detector (PSD) of $4^{\circ}$ of angular range.

Monochromatic $\mathrm{Cu} K_{\alpha}$ radiation was used. The electrooptic study was performed through texture observation in the polarizing microscope of samples prepared in commercially available cells (Linkam), of nominal thickness $5 \mu \mathrm{m}$. Electric fields of various amplitudes and frequencies were applied, and the birefringence was measured at different regions of the sample with a Berek compensator.

\section{RESULTS}

$\mathrm{X}$-ray measurements were carried out on cooling from the isotropic phase down to the low-temperature $\mathrm{SmC}_{A} \mathrm{P}_{A}$ phase. Figure 2 represents the small-angle $\mathrm{X}$-ray diagram obtained 
(1)

$\mathbf{I}-160^{\circ} \mathrm{C}(20.2)-\mathbf{B}_{1^{-1}} 120^{\circ} \mathrm{C}(0.5)-\mathbf{B}_{2^{-}}-83^{\circ} \mathrm{C}(13.9)$-Crystal
FIG. 1. Chemical structure and phase sequence on cooling of the studied compound. I represents the isotropic phase. The transition enthalpies, in brackets, are expressed in $\mathrm{kJ} / \mathrm{mol}$. at $140{ }^{\circ} \mathrm{C}$. Similar diagrams were obtained for different temperatures in the range $160-130{ }^{\circ} \mathrm{C}$. In all the cases the peaks were indexed on the basis of a rectangular lattice, assigning the $(h l)$ indices shown in Fig. 2. The fact that the lattice is rectangular irrespective of temperature means that its geometry is imposed by the symmetry of the structure, and cannot be a mere accidental situation that is usually temperature dependent. The structure is therefore orthorhombic, and previously reported models $[5,15,21,22]$ displaying monoclinic symmetry, although with rectangular translation lattices, must be discarded for our compound.

The temperature dependence of the observed reflections in the $h l$ reciprocal plane is schematized in Fig. 3. Our results indicate that the reciprocal lattice vector $c^{*}$ is constant in the whole temperature range of the $B_{1}$ phase [the Bragg angle of the (02) reflection does not change]. The cell parameter results $c=53.9 \AA$. On the other hand, $\boldsymbol{a}^{*}$ decreases continuously on cooling. The cell parameter $a$ increases from $77.3 \AA$ at $160^{\circ} \mathrm{C}$ to $90.7 \AA$ at $120^{\circ} \mathrm{C}$. The phase transition into the $\mathrm{SmC}_{A} \mathrm{P}_{A}$ phase, whose diffraction diagram is schematized by the solid dots in Fig. 3(c), occurs at about $120^{\circ} \mathrm{C}$. The temperature dependence of $a, c$, and the smectic layer spacing of the $\mathrm{SmC}_{A} \mathrm{P}_{A}$ phase, is represented in Fig. 4. A clear superposition of diffraction peaks from both phases is present at the transition temperature. In principle, even when this fact indicates a discontinuous transition, it could be also originated by a temperature gradient at the sample. The character of the $B_{1}-\mathrm{SmC}_{A} \mathrm{P}_{A}$ phase transition will be discussed in the next section.

From our x-ray diagrams the following aspects can be pointed out: the structure presents an orthorhombic symmetry and the $(h l)$ reflection indices satisfy the systematic

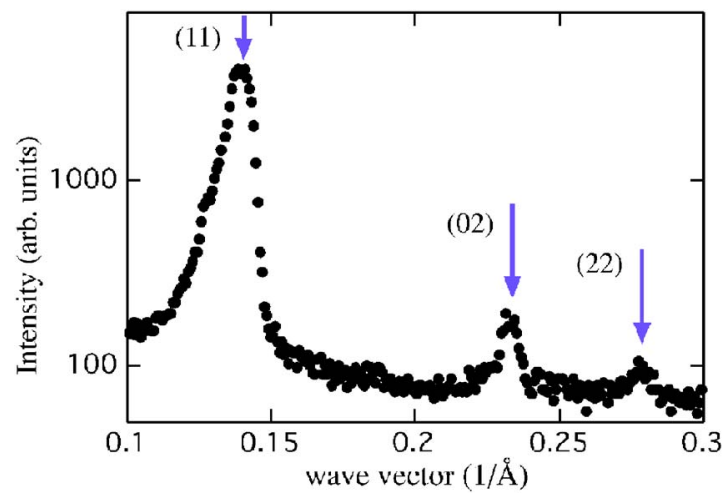

FIG. 2. (Color online) X-ray diffraction intensity as a function of the wave vector $q=4 \pi / \lambda \sin \theta_{B}$, where $\lambda$ is the wavelength and $\theta_{B}$ is the Bragg angle, for the $B_{1}$ phase of the studied material. Three peaks are observed for $\theta_{B}<2^{\circ}$, which were indexed as shown in the figure. extinction rule $h+l=2 n$. For nonresonant x-ray measurements, as is our case, the diffraction diagram corresponds to a lattice of columns where the molecules are projected on the lattice plane. Thus, the above extinction rule indicates that the translation lattice is centered. Two different structural models have been proposed to account for these conditions (see Fig. 5). They are antiferroelectric columnar phases with rectangular translation lattices, and have been extensively considered in the literature [16-19,22-24]. In both cases the $c$ parameter is just the molecular length. The first of these models was proposed by Watanabe et al. [16] and is normally known simply as $B_{1}$ (or conventional $B_{1}$ ) phase [Fig. 5(a)]. The molecules pack in such a way that their dipole moments are parallel to the lattice plane $a c$. The other model is the $B_{1}$ reversed ( $B_{1 \text { rev }}$ ) phase [Fig. 5(b)], and was proposed by Szydlowska et al. [19]. In this model the dipoles are perpendicular to the translation lattice.

We now analyze both models in more detail in order to decide if they are in agreement with our x-ray diagrams. Concerning the conventional $B_{1}$ structure, an important aspect to be pointed out is that the translation lattice of columns is not centered. This is a consequence of the geometry of the cross section of the columns, which are oriented differently at the origin and at the cell center [see Fig. 5(a)]. This situation does not occur in the case of the $B_{1 \text { rev }}$ structure, where the lattice of columns is strictly centered, and therefore the structure factor results

$$
F(h l)=f_{c}(h l)\left[1+e^{\pi i(h+l)}\right]
$$

where $f_{c}(h l)$ is the Fourier transform of the charge density of a given column. However, one problem arises: If the structure of Fig. 5(b) is considered, the charge density $\rho$ inside each column should depend only on the $z$ coordinate. This
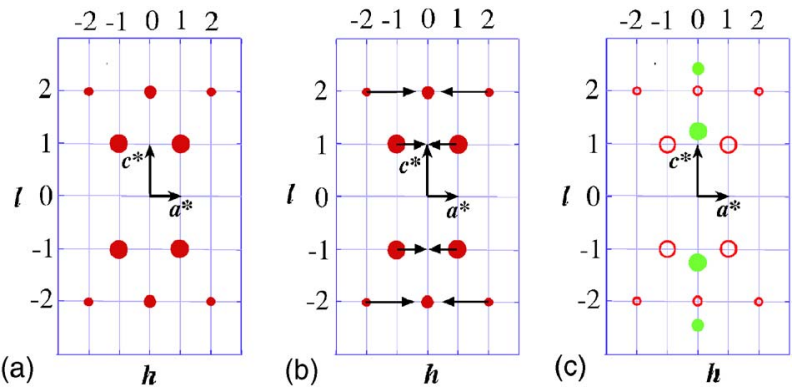

FIG. 3. (Color online) (a) Scheme of the $h l$ reciprocal plane where the observed reflections in the $B_{1}$ phase are indicated by dots. (b) On decreasing temperature $c^{*}$ is constant and $a^{*}$ diminishes. This trend is represented by the arrows. The solid dots in (c) represent the diffraction diagram of the low-temperature $\mathrm{SmC}_{A} \mathrm{P}_{A}$ phase. The missed reflections are indicated by open circles. 


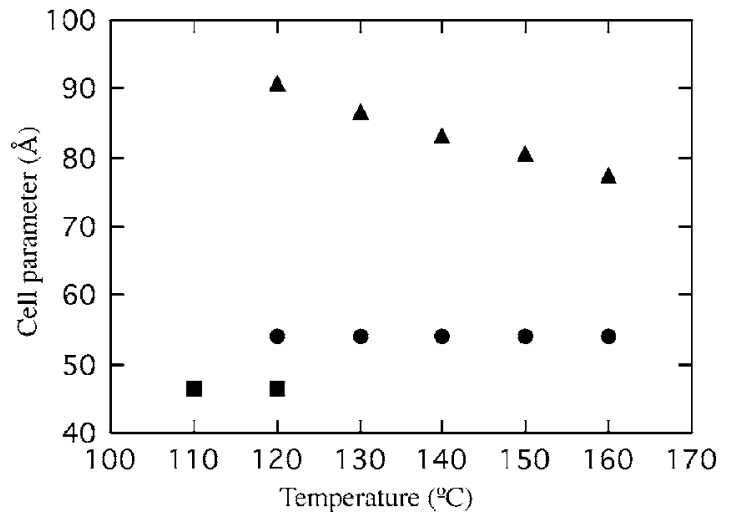

FIG. 4. Temperature dependence of the cell parameters $a$ (triangles) and $c$ (circles) of the $B_{1}$ phase, and the smectic layer spacing of the $\mathrm{SmC}_{A} \mathrm{P}_{A}$ phase (squares). A clear coexistence of both phases was detected at the transition temperature.

fact is a consequence of the liquid disorder inside the columns. Thus, since the columns width is just $a / 2, f_{c}(h l)$ for a column that extends from $x_{0}$ to $x_{0}+1 / 2$ (in units of $a$ ), and $z_{0}$ to $z_{0}+1$ (in units of $c$ ) is

$$
\begin{aligned}
f_{c}(h l) & =\int_{z_{0}}^{z_{0}+1} \int_{x_{o}}^{x_{o}+1 / 2} \rho(z) e^{2 \pi i(h x+l z)} d x d z \\
& =\left[\frac{e^{2 \pi i h x_{0}}}{2 \pi i h}\left[e^{\pi i h}-1\right]\right] \int_{z_{0}}^{z_{0}+1} \rho(z) e^{2 \pi i l z} d z,
\end{aligned}
$$

i.e., it is zero for $h=2,4,6, \ldots$, and, therefore, the (22) reflection that we observe in our diffraction diagrams should not be present. This inconsistency would imply either a column width different from $a / 2$ or an explicit dependence of $\rho$ both on $z$ and $x$ coordinates. This can only occur if there are packing defects (empty regions or superposition of columns), or splay of the polarization at the columns.

In summary, the structural models represented in Fig. 5 do not account for the diffraction diagrams that were obtained in the material under study, and a different model is therefore required. A discussion of different possibilities is presented in the next section.

The X-ray data above were complemented with a study of the optical textures to try to deduce a structural model for the $B_{1}$ phase. Figure 6 shows some selected textures obtained under the polarizing microscope. On slow cooling from the isotropic phase, the $B_{1}$ phase presents circular domains of purple color $(\Delta n=0.11)$ with the extinction brushes parallel

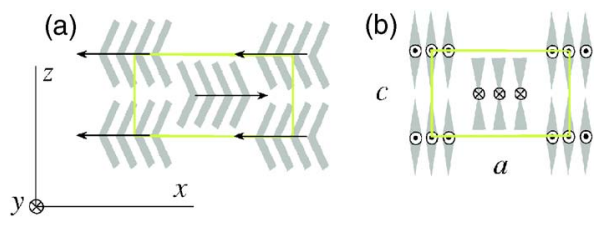

FIG. 5. (Color online) Structural models proposed for the conventional $B_{1}$ phase (a) and $B_{1 \text { rev }}$ phase (b). The rectangle represents the crystallographic cell, and the arrows indicate the polarization of each column.
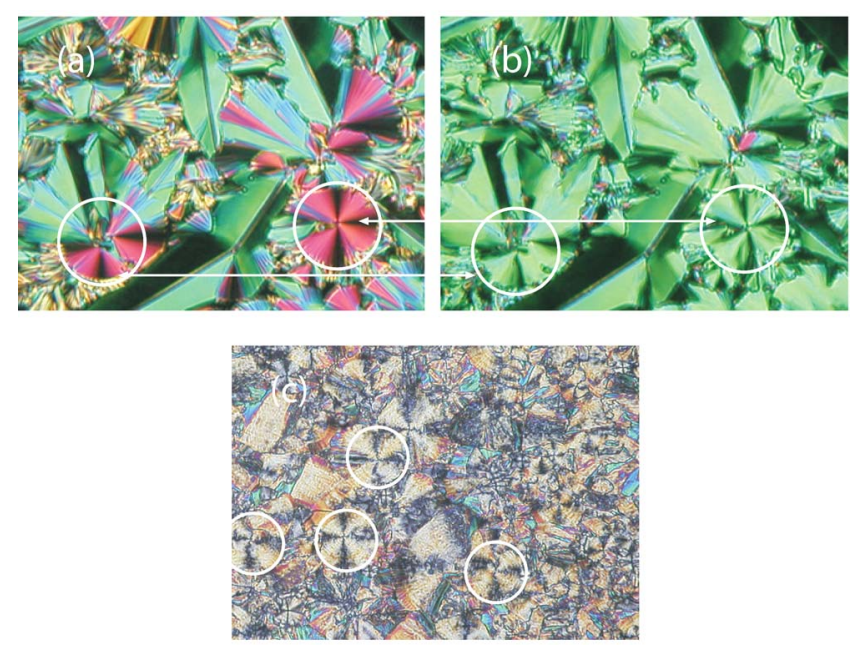

FIG. 6. (Color online) (a) Textures of the studied compound in the columnar phase on slow cooling under no field. The green banana-leaf domains present the maximum birefringence. (b) Under a square-wave electric field of $15 \mathrm{~V} \mathrm{\mu m}^{-1}$ the texture becomes green (with maximum birefringence) as a consequence of the dielectric anisotropy. The extinction brushes of the circular domains (see white circles) remain unaltered and along the polarizer directions. This fact indicates that the structure is nontilted (width of the photographs $200 \mu \mathrm{m}$ ). (c) Texture of the $\mathrm{SmC}_{A} \mathrm{P}_{A}$ phase obtained on cooling from the virgin columnar phase. The purple circular domains in the columnar phase transform into the yellowish circular domains (see white circles) below the transition. In the latter domains the smectic layers are in a bookshelf disposition with the molecular dipolar moments parallel to the substrates (width of the photograph $300 \mu \mathrm{m})$.

to the polarizer and analyzer directions [Fig. 6(a)]. Some green banana-leaf domains are also visible, with higher birefringence $(\Delta n=0.16)$. If a square-wave electric field of frequency $100 \mathrm{~Hz}$ and amplitude $15 \mathrm{~V} / \mu \mathrm{m}$ is applied, the purple domains change their color into green $(\Delta n=0.16)$ but the extinction directions are maintained [Fig. 6(b)]. With still larger fields $(>25 \mathrm{~V} / \mu \mathrm{m})$ the transition to $B_{2}$ takes place, and circular domains of green color $(\Delta n=0.16)$ with extinction directions at about $45^{\circ}$ appear (not shown in the figure) [13]. These domains are not correlated with any other domain in the $B_{1}$ phase. At lower temperatures, this material presents a $B_{2}$ ground state that under field exhibits the same texture than the $B_{2}$ phase obtained from $B_{1}$ by application of a strong field. On terminating the field, the green circular domains in the $B_{2}$ phase transform into gray color $(\Delta n$ $<0.02$ ) with vertical and horizontal extinction directions (not shown in Fig. 6) [13]. This clearly means that the $B_{2}$ phase is $\mathrm{SmC}_{A} \mathrm{P}_{A}$ without field and $\mathrm{SmC}_{S} \mathrm{P}_{F}$ under field. Finally, if the $B_{1}$ phase is cooled down without any field the shape of the purple domains and the extinction directions are maintained in the smectic phase, but present now a yellowish white color [Fig. 6(c)], with a birefringence $\Delta n=0.06$.

\section{DISCUSSION}

Now it is interesting to comment the experimental results described above in order to explain the structure of the co- 
lumnar phase. According to the texture observations, some important conclusions can be drawn: The birefringence of the $\mathrm{SmC}_{S} \mathrm{P}_{F}$ is the same as that of the banana-leaf domains [Fig. 6(a)], or the green circular domains under field in the columnar phase [Fig. 6(b)]. This fact implies that the structure of the green domains in the columnar phase is synclinic with the molecular dipole moments perpendicular to the substrate, as is the case in the $\operatorname{SmC}_{S} \mathrm{P}_{F}$ structure. Therefore, as the extinction brushes of the green circular domains in Fig. 6(b) are parallel to the polarizers, the structure must be nontilted.

This conclusion is compatible with the orthorhombic symmetry deduced from the $\mathrm{x}$-ray measurements. However, one point deserves some comment. The value of the $c$ parameter obtained for this phase is not compatible with a nontilted structure in which the molecules are in the most extended configuration (the calculated molecular length is about 15\% larger than $c$ ). This fact is in contrast with what is usual in banana-type mesophases, where the smectic and molecular lengths are usually compatible with the tilt. There are two possible explanations to this discrepancy: interdigitation or terminal chain disorder [25]. In both cases a reduction of the effective chain length takes place, which would allow the core-core interaction between adjacent ribbons to compete with the core-chain segregation tendency. This interaction competition is believed to be essential for the existence of columnar phases [7,26]. In fact, Shen et al. [7] studied the phase sequences of a family of compounds with the same core as the compound of the present work and found that in compounds with alkoxy terminal chains the columnar phases were present up to 11 carbon atoms. In addition, in that work, an increasing mismatch between the molecular length and $c$ parameter was observed as the number of carbon atoms in the terminal chain increases. This fact would also imply interdigitation or chain disorder. In our compound the terminal chain is longer (14 carbon atoms), and therefore, this effect is expected to be much more pronounced.

The study of the temperature dependence of the columnar phase as the transition is approached can be also helpful for the understanding of the columnar phase structure. Two experimental aspects should be pointed out.

(1) According to DSC results the phase transition is weakly first order but definitely discontinuous. This conclusion is based on the existence of a small but non-null transition enthalpy $(0.5 \mathrm{~kJ} / \mathrm{mol})$ and rather remarkable transitiontemperature hysteresis $\left(\Delta T=10^{\circ} \mathrm{C}\right)$. Both values have been obtained from the extrapolation to zero deduced from different heating and cooling rates. However, the transition enthalpy is quite small for bent-core liquid-crystal standards. This fact indicates that the energy cost for the phase transition must be low and therefore the molecular rearrangement that takes place in the process must not be very drastic. This point is especially important in this case since the hightemperature mesophase is nontilted whereas the $\mathrm{SmC}_{A} \mathrm{P}_{A}$ presents a tilt of $42^{\circ}$. The order of magnitude of the enthalpy cost for the tilting process can be estimated from the results obtained by Wirth et al. [27] in several banana compounds in a first-order transition $\mathrm{Sm}_{A}-\mathrm{Sm}_{C}$. In all cases the obtained enthalpies have the same order of magnitude than that of the transition in our compound. This fact implies that the process of layer formation at the transition cannot take place with large energy requirements.

(2) The length of the lattice vector $\boldsymbol{a}$ increases as the temperature approaches the transition. This effect could be considered as a trend of the phase towards the $\mathrm{SmC}_{A} \mathrm{P}_{A}$ structure. This point can be easily understood if we consider the smectic phase as a limit case of the columnar one in which the lattice vector $\boldsymbol{a}$ becomes infinite. This behavior has been also observed in other compounds presenting phase transitions from two-dimensional (2D) undulated structures to SmCP lamellar phases $[20,28,29]$.

As stated above, the columnar phase is orthorhombic, nontilted and, for nonresonant x-rays, centered. Additionally, this phase has been reported to be antiferroelectric [13]. A ferroelectric ground state can be excluded because no second-harmonic generation can be observed in the material under no field. Figure 7(a) represents a proposed structure compatible with all those experimental features. In this model a splay of the polarization is incorporated which would allow for the existence of the x-ray reflection (22) (for simplicity in the figure a splay that implies a $180^{\circ}$ rotation of the dipole moments between the edges of the column has been drawn but a weaker splay is more likely). The existence of splay of the polarization in columnar phases has also been proposed by Coleman et al. [20]. As can be seen, the dipole moment distribution in adjacent ribbons implies a frustrated lamellar phase. Under this model the ribbon cross sections will elongate as the temperature decreases, up to a maximum length at which the columnar phase becomes unstable. At this point, half of the ribbons undergo a displacement of $c / 2$ along the $z$ direction with respect to the others to form the $\mathrm{SmC}_{A} \mathrm{P}_{A}$ phase. Although it is impossible to determine the polarization in adjacent ribbons by common $\mathrm{x}$-ray experiments, the proposed structure implies a less dramatic molecular rearrangement at the transition than other possibilities.

Figure 7(b) represents a scheme of the proposed structure where the symmetry elements are indicated. The layer group referred to the setting $(x, y, z)$ indicated in the figure is $p$ mca (layer group No. $45[30]$ ). The yellow rectangle is the same crystallographic cell as in Fig 7(a). The cell parameter along $z$ is twice the cell parameter $c$ obtained from the x-ray measurements. Since experimentally we cannot distinguish the polarization direction of the ribbons, the lattice of columns appears as rectangular centered with lattice parameters $a$ and $c$.

A final remark can be made regarding the different types of domains that the texture of the columnar phase exhibits. Figure 8 shows the most often found types of domains. As can be seen, the green domains are leaf-shaped whereas the purple ones are always circular with the extinction brushes parallel to the polarizers. As reported in Ref. [13], considering the molecule as formed by two uniaxial wings that form a bending angle of about $120^{\circ}$, with ordinary and extraordinary indices, respectively, $n_{o}=1.5, n_{e}=1.7$, the birefringence of both types of domains can be quantitatively explained. In the banana-leaf domains the birefringence is consistent with an orientation of the dipolar moments perpendicular to the substrate whereas in the purple domains, the molecules are in planar disposition with the dipolar moments parallel to the 

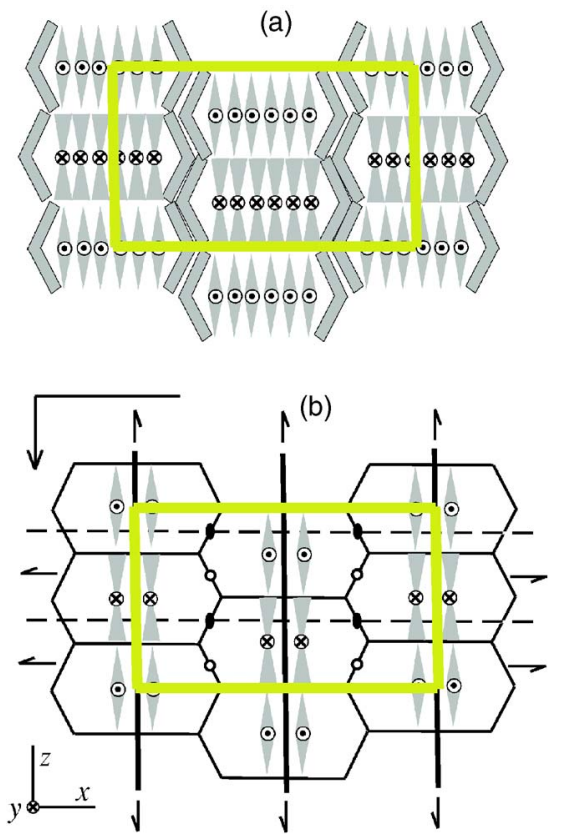

FIG. 7. (Color online) (a) Schematic structure for the columnar phase of the studied compound. The structure is a rectangular nontilted phase with splay of the polarization inside the columns (for simplicity in the figure a splay that implies a $180^{\circ}$ rotation of the dipole moments between the edges of the column has been drawn but this extreme is not a requirement and a weaker splay is expected). The dipolar moment disposition in adjacent layers can be understood as a frustration of a lamellar phase. (b) Schematic representation of the symmetry elements of the structure. The corresponding layer group is $p$ mca.

glass surfaces. Under this scheme the correlation between molecular orientation and domain shape can be explained on the basis of the structural model proposed for this phase. The situation is sketched in Fig. 8. In the banana-leaf domains the column axes are perpendicular to the substrate. Therefore this orientation of the columns prevents the structure from forming circular domains. In contrast, in the purple domains the columnar axes are parallel to the substrate allowing the domains to grow in circular shape to minimize the energy of the domain walls. The molecular disposition in the circular domains is also in agreement with the texture that they present in the $\mathrm{SmC}_{A} \mathrm{P}_{A}$ phase. As shown in Fig. 6(c), on cooling from the columnar phase without field down to the $\mathrm{SmC}_{A} \mathrm{P}_{A}$ structure, the circular domains remain but they present now a yellowish color with smaller birefringence $\Delta n=0.06$. This value is consistent with a planar domain wherein the molecules are oriented with the dipole moments parallel to the substrate, as is the case in those domains in the parent phase. In fact, under the birefringence calculation scheme explained above and using the same parameters together with the corresponding tilt of the $\operatorname{SmC}_{A} \mathrm{P}_{A}$ phase $\left(42^{\circ}\right)$, a very good agreement is obtained also for the birefringence of the yellowish domains (calculated birefringence $\Delta n=0.06)$.

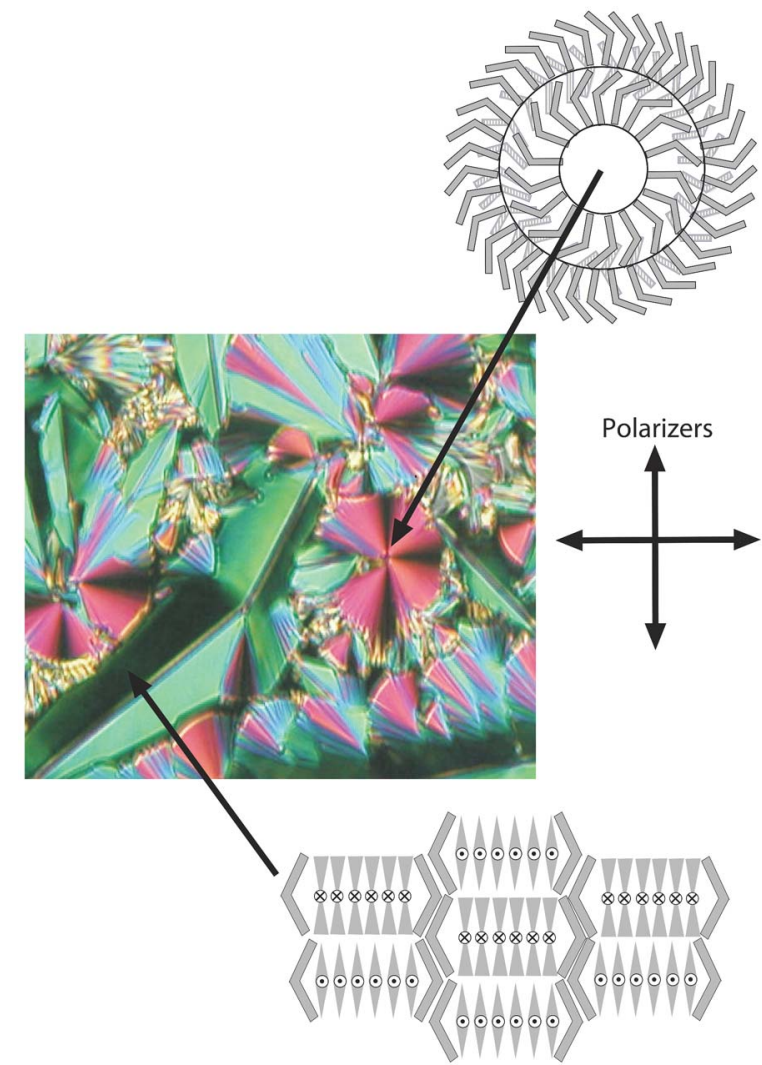

FIG. 8. (Color online) The most representative types of domains in the columnar phase. This texture is obtained on slow cooling from the isotropic phase under no field. The molecular disposition in the banana-leaf and circular domains appears schematized in the figure. The width of the photograph is $150 \mu \mathrm{m}$.

In summary, we have studied a columnar to smectic transition by means of $\mathrm{x}$-ray measurements as a function of the temperature. The columnar structure is similar to the $B_{1 \text { rev }}$ phase with the addition of some splay of the polarization. On decreasing the temperature the length of the columns cross section increases and, at the transition point, a displacement of the molecules along the layer normal in every second column takes place. The transition is clearly first order though the enthalpy content is surprisingly small. It would be interesting to carry out studies in other materials to confirm whether this transitional behavior is general in the columnar to $\mathrm{SmCP}$ transition or not. Unfortunately, very few materials exhibiting this kind of phase transition have been reported and, apart from the present work, studies of the evolution of the structure with the temperature are scarce.

\section{ACKNOWLEDGMENTS}

This work was supported by the CICYT-FEDER of Spain-EU (Project No. MAT2003-07806-C02), the University of the Basque Country (Project No. 9/UPV 00060.310-13562/2001), and the Aragón Government. 
[1] G. Pelzl, S. Diele, and W. Weissflog, Adv. Mater. (Weinheim, Ger.) 11, 707 (1999).

[2] C. Tschierske and G. Dantlgraber, Pramana, J. Phys. 61, 455 (2003).

[3] M. B. Ros, J. L. Serrano, M. R. de la Fuente, and C. L. Folcia, J. Mater. Chem. 15, 5093 (2005).

[4] H. Takezoe and Y. Takanishi, Jpn. J. Appl. Phys., Part 1 45, 597 (2006).

[5] R. Amaranatha Reddy and C. Tschierske, J. Mater. Chem. 16, 907 (2006).

[6] D. R. Link, G. Natale, R. Shao, J. E. Maclennan, N. A. Clark, E. Körblova, and D. M. Walba, Science 278, 1924 (1997).

[7] D. Shen, A. Pegenau, S. Diele, I. Wirth, and C. Tschierske, J. Am. Chem. Soc. 122, 1593 (2000).

[8] H. N. Shreenivasa Murthy and B. K. Sadashiva, Liq. Cryst. 29, 1223 (2002).

[9] J. C. Rouillon, J. P. Marcerou, M. Laguerre, H. T. Nguyen, and M. F. Achard, J. Mater. Chem. 11, 2946 (2001).

[10] W. Weissflog, F. Wirth, S. Diele, G. Pelzl, H. Schmalfuss, T. Schoss, and A. Würflinger, Liq. Cryst. 28, 1603 (2001).

[11] G. Dantlgraber, D. Shen, S. Diele, and C. Tschierske, Chem. Mater. 14, 1149 (2002).

[12] H. N. Shreenivasa Murthy and B. K. Sadashiva, Liq. Cryst. 31, 1347 (2004).

[13] J. Ortega, M. R. de la Fuente, J. Etxebarria, C. L. Folcia, S. Díez, J. A. Gallastegui, N. Gimeno, M. B. Ros, and M. A. Pérez-Jubindo, Phys. Rev. E 69, 011703 (2004).

[14] H. N. Shreenivasa Murthy, M. Bodyagin, S. Diele, U. Baumeister, G. Pelzl, and W. Weissflog, J. Mater. Chem. (unpublished).

[15] R. Amaranatha Reddy, V. A. Raghunathan, and B. K. Sadashiva, Chem. Mater. 17, 274 (2005).

[16] J. Watanabe, T. Niori, T. Sekine, and H. Takezoe, Jpn. J. Appl. Phys., Part 1 37, L139 (1998).
[17] Y. Takanishi, T. Izumi, J. Watanabe, K. Ishikawa, H. Takezoe, and A. Iida, J. Mater. Chem. 9, 2771 (1999).

[18] Y. Takanishi, H. Takezoe, J. Watanabe, Y. Takahashi, and A. Iida, J. Mater. Chem. 16, 816 (2006).

[19] J. Szydlowska, J. Mieczkowski, J. Matraszek, D. W. Bruce, E. Gorecka, D. Pociecha, and D. Guillon, Phys. Rev. E 67, 031702 (2003).

[20] D. A. Coleman, J. Fernsler, N. Chattham, M. Nakata, Y. Takanishi, E. Körblova, D. R. Link, R. F. Shao, W. G. Jang, J. E. Maclennan, O. Mondainn-Mova, C. Boyer, W. Weissflog, G. Pelzl, L. C. Chien, J. Zasadzinski, J. Watanabe, D. M. Walba, H. Takezoe, and N. A. Clark, Science 301, 1204 (2003).

[21] C. Keith, R. Amaranatha Reddy, U. Baumeister, and C. Tschierske, J. Am. Chem. Soc. 126, 14313 (2004).

[22] R. Amaranatha Reddy, B. K. Sadashiva, and V. A. Raghunathan, Chem. Mater. 16, 4050 (2004).

[23] K. Pelz, W. Weissflog, U. Baumeister, and S. Diele, Liq. Cryst. 30, 1151 (2003).

[24] H. T. Nguyen, J. P. Bedel, J. C. Rouillon, J. P. Marcerou, and M. F. Achard, Pramana, J. Phys. 61, 395 (2003).

[25] A. Eremin, H. Nadasi, G. Pelzl, S. Diele, H. Kresse, W. Weissflog, and S. Grande, Phys. Chem. Chem. Phys. 6, 1290 (2004).

[26] P. Pratibha, N. V. Madhusudana, and B. K. Sadashiva, Pramana, J. Phys. 61, 405 (2003).

[27] I. Wirth, S. Diele, A. Eremin, G. Pelzl, S. Grande, L. Kovalenko, N. Pancenko, and W. Weissflog, J. Mater. Chem. 11, 1642 (2001).

[28] S. Kang, M. Tokita, K. Ogino, T. Doi, T. Takahashi, H. Takezoe, and J. Watanabe, Phys. Rev. E 73, 011701 (2006).

[29] C. L. Folcia, I. Alonso, J. Ortega, J. Etxebarria, I. Pintre, and M. B. Ros, Chem. Mater. (to be published).

[30] International Tables for Crystallography, edited by V. Kopsky and D. B. Litvin (Kluwer Academic Publishers, Dordrecht, 2002), Vol. E. 